\title{
Remarks on the Limiting Gibbs States on a $(d+1)$-Tree
}

By

\section{Yasunari HIGUCHI*}

\section{§1. Introduction}

In this paper we investigate limiting Gibbs states with nearest neighbour ferromagnetic potentials on a $(d+1)$-tree. Preston [1] has got a necessary and sufficient condition for the non-uniqueness of the Gibbs states for a given interaction (i.e. the necessary and sufficient condition for the phase transition to occur) in these models. The Gibbs states on a countable tree are studied in [1] and [2]. Our aim is to obtain several limiting Gibbs states by changing boundary conditions.

Spitzer [2] has shown that (i) every extremal Gibbs state invariant under graph isomorphisms is a "Markov chain" in the sense of his definition (see Definition 1), and (ii) there are at most three "Markov chains" among the Gibbs states for any given nearest neighbour ferromagnetic potential. In section 4 we will prove that every "Markov chain" which is Gibbsian for the given interaction is obtained as a limiting Gibbs state for the same interaction with certain boundary conditions.

In section 5 we will give examples of limiting Gibbs states such that the number of up-spins appearing in the corresponding boundary conditions is much smaller than that of down-spins on every boundary, while the probability for the spin at the origin to be up is larger than $1 / 2$.

In section 6 we will give several extremal Gibbs states using above examples.

Communicated by K. Itô, June 18, 1976.

* Department of Mathematics, Kyoto University, Kyoto, 606 Japan.

Presnt Address: Department of Mathematics, Kobe University, Kobe, 657 Japan. 
Section 2 and 3 are devoted to the preliminary definitions and discussions.

\section{§2. Definitions and Notations}

Let $T$ be a $(d+1)$-tree with a positive integer $d \geqq 2$. Two points $t, s \in T(t \neq s)$ are called to be neighbours if they are connected by one bond. Each point of $T$ has $d+1$ neighbours. We fix an origin 0 of $T$. We write $t \rightarrow s$ if $t \neq s$ and the path connecting 0 and $s$ passes $t$. If $t \rightarrow s$ and $t, s$ are neighbours, we write $t \rightarrow s$. For any finite $V \subset T$, the boundary $\partial V$ of $V$ is

$$
\partial V=\{t \in T \backslash V ; \text { there exists a neighbour of } t \text { in } V\} .
$$

For every $V \subset T$, let $\Sigma_{V}=\{-1,+1\}^{V}$ be the space of all possible spin configurations on $V$. In particular we write $\Sigma$ instead of $\Sigma_{T}$. We assign the product topology to $\Sigma$. For every $V \subset T$, define the $\sigma$-algebra $\mathscr{B}_{V}$ by

$$
\mathscr{B}_{V}=\text { the } \sigma \text {-algebra generated by }\left\{X_{t}, t \in V\right\} \text {, }
$$

where $X_{t}(\sigma)=\sigma(t)$ for all $t \in T, \sigma \in \Sigma$. In particular we write $\mathscr{B}$ instead of $\mathscr{B}_{T}$.

Let $V$ be a finite subset of $T, \omega \in \Sigma$ and $\sigma \in \Sigma_{V}$. We define an interaction energy on $V$ with the inner configuration $\sigma$ and the boundary condition $\omega$ by

$$
\begin{aligned}
E_{V}^{\omega}(\sigma)= & -H \sum_{t \in V} \sigma(t)-J \sum_{t, s \in V ; t \rightarrow s} \sigma(t) \sigma(s) \\
& -J \sum_{t \in V, s \in \partial V ; t, s \text { are neighbours }} \sigma(t) \omega(s),
\end{aligned}
$$

where $H$ is a real number called the external magnetic field and $J$ is a positive number called the ferromagnetic nearest neighbour potential. A finite Gibbs state $P_{V}^{\omega}$ on $\Sigma_{V}$ corresponding to $E_{V}^{\omega}$ is defined by

$$
P_{V}^{\omega}(\sigma)=\left[Z_{V}^{\omega}\right]^{-1} \exp \left[-E_{V}^{\omega}(\sigma)\right] \quad \sigma \in \Sigma_{V}
$$

where $Z_{V}^{\omega}=\sum_{\hat{\sigma} \in \Sigma_{V}} \exp \left[-E_{V}^{\omega}(\hat{\sigma})\right]$. As usual, $P_{V}^{\omega}$ can be considered as a probability measure on $(\Sigma, \mathscr{B})$. 
For fixed $H$ and $J$, if there is an increasing sequence of finite subsets $\left\{V_{n}\right\}$ such that $V_{n} \nearrow T$ as $n \rightarrow \infty$ and $P^{\omega}=\mathrm{w}-\lim _{n \rightarrow \infty} P_{V_{n}}^{\omega}$ (the weak convergence of measures) exists for suitably fixed $\omega \in \Sigma$, then $P^{\omega}$ is called a limiting Gibbs state with boundary condition $\omega$ for $(H, J)$. On the other hand, a Gibbs state $P$ for $(H, J)$ is defined as a probability measure on $(\Sigma, \mathscr{B})$ such that for every $A$ in $\mathscr{B}_{V}$,

$$
P\left(A \mid \mathscr{B}_{V^{c}}\right)(\omega)=P_{V}^{\omega}(A) \quad \text { a.s. } \quad(P)
$$

It is well known that the set $\mathscr{G}(H, J)$ of all Gibbs states for any fixed $(H, J)$ is a non-empty, compact convex set. A limiting Gibbs state is a Gibbs state for the same $(H, J)$. Conversely, every extremal point of $\mathscr{G}(H, J)$ is a limiting Gibbs state with a suitable boundary condition for the same $(H, J)$.

\section{§3. Limiting Gibbs States}

Let $|t|$ denote the distance between 0 and $t \in T$, i.e. $|t|=n$ if there exists a chain $0 \rightarrow u_{1} \rightarrow u_{2} \rightarrow \cdots \overrightarrow{1} u_{n-1} \rightarrow t$. From now on we only consider the sequence of boxes $V_{n}=\{t \in T ;|t| \leqq n\}, n \geqq 1$. For every $s \in T$ we define $T_{s}=\{s\} \cup\{t \in T ; s \rightarrow t\}$ and $V_{n, s}=T_{s} \cap V_{n}, n \geqq 1$. The members of $V_{1} \backslash\{0\}$ are numbered by $1,2, \ldots, d+1$. For every $\omega \in \Sigma, s \in T \backslash\{0\}$ and $n \geqq|s|$, put

$$
\begin{gathered}
W_{n, s}^{\omega}(\varepsilon)=\sum_{\sigma \in \Sigma_{V}, s ; \sigma(s)=\varepsilon} \exp \left[-E_{V_{n, s}}^{\omega}(\sigma)-J \varepsilon \omega(t)\right], \quad \varepsilon= \pm 1 \\
R_{n, s}(\omega)=W_{n, s}^{\omega}(+1) / W_{n, s}^{\omega}(-1)
\end{gathered}
$$

where $t$ is the unique point such that $t \rightarrow s$. Then we get

$$
\begin{gathered}
P_{V_{n}}^{\omega}(\sigma(0)=+1) / P_{V_{n}}^{\omega}(\sigma(0)=-1) \\
=e^{2 H} \prod_{i=1}^{d+1}\left[e^{J} R_{n, i}(\omega)+e^{-J}\right] /\left[e^{-J} R_{n, i}(\omega)+e^{J}\right], \\
W_{n, s}^{\omega}(\varepsilon)=e^{\varepsilon H} \prod_{u ; s \rightarrow \mathfrak{i} u}\left[e^{\varepsilon J} W_{n, u}^{\omega}(+1)+e^{-\varepsilon J} W_{n, u}^{\omega}(-1)\right], \quad \varepsilon= \pm 1
\end{gathered}
$$

and for $n>m, \eta \in \Sigma_{V_{m}}$, 


$$
\begin{aligned}
P_{V_{n}}^{\omega}\left(\left\{\sigma(s)=\eta(s), s \in V_{m}\right\}\right) \\
=\frac{\exp \left[-E_{V_{m-1}}^{\eta}(\eta)\right] \prod_{s \in \partial V_{m-1}} W_{n, s}^{\omega}(\eta(s))}{\sum_{\xi \in \Sigma_{V m}} \exp \left[-E_{V_{m-1}}^{\xi}(\xi)\right] \prod_{s \in \partial V_{m-1}} W_{n, s}^{\omega}(\xi(s))} .
\end{aligned}
$$

From the above equalities we obtain easily the following

Lemma 1. Let $\omega \in \Sigma$ be given. If there is $N>0$ such that $R_{n, s}(\omega)$ converges as $n \rightarrow \infty$ for every $s \in T \backslash V_{N}$, then $P^{\omega}=\mathrm{w}-\lim _{n \rightarrow \infty} P_{V_{n}}^{\omega}$ exists.

Now, let us consider a boundary condition $\omega$ such that $c(\omega)=$ $\sum_{s ; t \rightarrow s} \omega(s)$ is independent of $t \in T /\{0\}$. Then it is easy to see that $R_{n}(\omega)$ $=R_{n, i}(\omega)$ is independent of $i=1,2, \ldots, d+1$, and $R_{n, s}(\omega)=R_{n-|s|+1}(\omega)$. Hence we get

$$
Y_{n}(\omega)=H+d f\left(Y_{n-1}(\omega)\right)
$$

from (7), where $Y_{n}(\omega)=\frac{1}{2} \log R_{n}(\omega)$, and

$$
f(x)=\operatorname{th}^{-1}(\operatorname{th} J \cdot \operatorname{th} x)=\frac{1}{2} \log \left[\frac{\operatorname{ch}(x+J)}{\operatorname{ch}(x-J)}\right],
$$

which is a concave increasing function on $[0, \infty)$ and $f(-x)=-f(x)$ for all $x$ (see [1], [3]).

The following is obtained from (9) and FKG inequality.

Theorem (Preston [1]). For fixed $(H, J)$, the following (i) (iii) are equivalent:

(i) There exist two different Gibbs states (i.e. a phase transition occurs).

(ii) The equation

$$
x=H+d f(x)
$$

has more than one solutions.

(iii) $J>\frac{1}{2} \log \left[\frac{d+1}{d-1}\right]$ and $|H| \leqq d f(u)-u$,

where $u$ is the unique positive value such that $f^{\prime}(u)=1 / d$. 
The equation (11) has at most three solutions, say $\alpha \leqq \beta \leqq \gamma$. Preston [1] has shown that $Y_{n}\left(\omega^{+}\right) \searrow \gamma$ and $Y_{n}\left(\omega^{-}\right) / \alpha$ as $n \rightarrow \infty$, where $\omega^{+}, \omega^{-} \in \Sigma$ are defined by

$$
\omega^{+}(t)=+1, \omega^{-}(t)=-1 \quad \text { for all } t \in T .
$$

Since (9) implies that the asymptotic behavior of $Y_{n}(\omega)$ depends only on $Y_{1}(\omega)$, we get

$$
\lim _{n \rightarrow \infty} Y_{n}(\omega)=\left\{\begin{array}{lll}
\alpha & \text { if } & H+J c(\omega)<\beta \\
(\beta & \text { if } & H+J c(\omega)=\beta) \\
\gamma & \text { if } & H+J c(\omega)>\beta
\end{array}\right.
$$

Combining Lemma 1 and (13), we have

$$
P^{\omega}=\left\{\begin{array}{lll}
P^{\omega^{+}} & \text {if } & H+J c(\omega)>\beta \\
P^{\omega^{-}} & \text {if } & H+J c(\omega)<\beta
\end{array}\right.
$$

In general, $c_{t}(\omega)=\sum_{s ; t \rightarrow s} \omega(s)$ depends on $t \in T \backslash\{0\}$, but from FKG inequality we have

Proposition 1. For a general $\omega \in \Sigma$,

$$
P^{\omega}=\left\{\begin{array}{lllll}
P^{\omega^{+}} & \text {if } & H+J c_{t}(\omega)>\beta & \text { for all } & t \in T \backslash\{0\}, \\
P^{\omega^{-}} & \text {if } & H+J c_{t}(\omega)<\beta & \text { for all } & t \in T \backslash\{0\}
\end{array}\right.
$$

\section{§4. Markov Chains}

In this section we construct the third "Markov chain".

Definition 1 (Spitzer [2]). A probability measure $P$ on $(\Sigma, \mathscr{B})$ is called a "Markov chain" if there is a $2 \times 2$ positive stochastic matrix $Q$ with its invariant probability measure $\pi(\pi Q=\pi)$ such that for any connected finite subset $V$ of $T$ and for any $\varepsilon \in \Sigma_{V}$,

$$
P(\{\sigma(t)=\varepsilon(t), t \in V\})=\pi\left(\varepsilon\left(t_{0}\right)\right) \prod_{\substack{t, t^{\prime} \in V \\ t \overrightarrow{1}^{\prime}}} Q\left(\varepsilon(t), \varepsilon\left(t^{\prime}\right)\right),
$$

where $t_{0}$ is the point such that $t_{0} \in V$ and $t_{0} \rightarrow t$ for every $t \in V \backslash\left\{t_{0}\right\}$. 
We remark that the above definition does not depend on the choice of the origin because $(Q, \pi)$ is reversible.

Let $\mathscr{M}$ be the set of all "Markov chains"

Theorem (Spitzer [2]). (i) If $P$ is an extremal point of $\mathscr{G}(H, J)$ and invariant under graph isomorphisms (i.e. bijections of $T$ preserving the relation of neighbouring), then $P \in \mathscr{M}$.

(ii) There are at most three elements in $\mathscr{M} \cap \mathscr{G}(H, J)$ for every $(H, J)$.

Obviously $P^{\omega^{+}}$and $P^{\omega^{-}}$are "Markov chains" from the above theorem (i). As for the third element of $\mathscr{M} \cap \mathscr{G}(H, J)$, at first we consider the case when $H=0$ (then $\beta=0$ ) and $d$ is even. In this case, putting $\omega^{0}$ to satisfy $c_{t}\left(\omega^{0}\right)=0$ for all $t \in T \backslash\{0\}$, we have $\lim _{n \rightarrow \infty} R_{n, t}\left(\omega^{0}\right)=1$ for all $t \in$ $T \backslash\{0\}$. Hence from Lemma 1 and (6), we have the existence of $P^{\omega^{0}}$ different from $P^{\omega^{+}}$and $P^{\omega^{-}}$when $J>\frac{1}{2} \log [(d+1) /(d-1)]$. In general, we have

Theorem 1. Let $\alpha \leqq \beta \leqq \gamma$ be the possible solutions of (11). Then we have the followings:

(i) If three sequences $\left\{d_{n}\right\},\left\{a_{n}\right\},\left\{\tilde{a}_{n}\right\}$ of real numbers satisfy

(14-a) $\quad 1 \leqq d_{n} \leqq d, \quad d_{n}$ is an integer,

(14-b) $\quad a_{n}=H+d_{n} f\left(a_{n-1}\right)+\left(d-d_{n}\right) f\left(\tilde{a}_{n-1}\right) \geqq \beta$,

(14-c) $\quad \tilde{a}_{n}=H+\left(d_{n}-1\right) f\left(a_{n-1}\right)+\left(d-d_{n}+1\right) f\left(\tilde{a}_{n-1}\right)<\beta$,

for every $n \geqq 1$, then $\lim _{n \rightarrow \infty} a_{n}=\lim _{n \rightarrow \infty} \tilde{a}_{n}=\beta$.

(ii) Let us take the initial conditions;

$$
\begin{aligned}
& d_{1}=\min \{k ; k \text { is an integer and } H+(2 k-d) J \geqq \beta\}, \\
& a_{1}=H+\left(2 d_{1}-d\right) J, \quad \tilde{a}_{1}=H+\left(2 d_{1}-2-d\right) J .
\end{aligned}
$$

Then a triple of sequences $\left\{d_{n}\right\},\left\{a_{n}\right\},\left\{\tilde{a}_{n}\right\}$ can be defined inductively to satisfy (14). Moreover, there is a configuration $\omega^{0} \in \Sigma$ such that $Y_{n, s}\left(\omega^{0}\right)$ $=a_{n-|s|+1}$ or $\tilde{a}_{n-|s|+1}$ for every $s \in T \backslash\{0\}$, where 


$$
Y_{n, s}(\omega)=\frac{1}{2} \log R_{n, s}(\omega)
$$

(iii) The limiting Gibbs state $P^{\omega^{0}}$ exists and $\mathscr{M} \cap \mathscr{G}(H, J)=\left\{P^{\omega^{+}}\right.$, $\left.P^{\omega^{-}}, P^{\omega^{0}}\right\}$ for all $H$ and $J$.

Proof. (i) From (14) and the property of $f(x)$, we have $0<a_{n}-\tilde{a}_{n}$ $=f\left(a_{n-1}\right)-f\left(\tilde{a}_{n-1}\right) \leqq 2 f\left(\left(a_{n-1}-\tilde{a}_{n-1}\right) / 2\right)$. Since $f(x) \leqq x$ for $x \geqq 0$ and the equality holds if and only if $x=0$, we get $\lim _{n \rightarrow \infty}\left(a_{n}-\tilde{a}_{n}\right)=0$, which implies $\lim _{n \rightarrow \infty} a_{n}=\lim _{n \rightarrow \infty} \tilde{a}_{n}=\beta$.

(ii) The first part is easy to verify. To construct $\omega^{0}$, we define $\omega_{n}^{0}, \tilde{\omega}_{n}^{0} \in \Sigma_{\partial V_{n, 1} \backslash\{0\}}$ inductively. Let $\partial V_{1,1} \backslash\{0\}=\left\{s_{1}, \ldots, s_{d}\right\}$. We define $\omega_{1}^{0}$ and $\tilde{\omega}_{1}^{0}$ on $\partial V_{1,1} \backslash\{0\}$ as $\sum_{1 \leqq v \leqq d} \omega_{1}^{0}\left(s_{v}\right)=2 d_{1}-d$, and $\sum_{v=1}^{d} \tilde{\omega}_{1}^{0}\left(s_{v}\right)=2 d_{1}-d-2$. Let $\tau_{v}$ be a shift operator from $T_{s_{v}}$ to $T_{1}$ such that $\tau_{v}\left(s_{v}\right)=1 \in T_{1}$ for every $1 \leqq v \leqq d$. Then we can define $\omega_{n}^{0}$ and $\tilde{\omega}_{n}^{0}$ on $\partial V_{n, 1} \backslash\{0\}$ by

$$
\begin{aligned}
& \omega_{n}^{0}(t)=\left\{\begin{array}{llll}
\omega_{n-1}^{0}\left(\tau_{v}(t)\right) & \text { if } & t \in T_{s_{v}} \cap \partial V_{n}, & 1 \leqq \nu \leqq d_{n}, \\
\tilde{\omega}_{n-1}^{0}\left(\tau_{v}(t)\right) & \text { if } & t \in T_{s_{v}} \cap \partial V_{n}, & d_{n}+1 \leqq \nu \leqq d,
\end{array}\right. \\
& \tilde{\omega}_{n}^{0}(t)=\left\{\begin{array}{llll}
\omega_{n-1}^{0}\left(\tau_{v}(t)\right) & \text { if } & t \in T_{s_{v}} \cap \partial V_{n}, & 1 \leqq \nu \leqq d_{n}-1, \\
\tilde{\omega}_{n-1}^{0}\left(\tau_{v}(t)\right) & \text { if } & t \in T_{s_{v}} \cap \partial V_{n}, & d_{n} \leqq v \leqq d .
\end{array}\right.
\end{aligned}
$$

Now put $\omega^{0}(t)=\omega_{n}^{0}(t)$ for $t \in \partial V_{n, 1} \backslash\{0\}, n \geqq 1$ and extend it to be invariant under the rotations around the origin. Then it is easy to see that $\omega^{0}$ is a required configuration.

(iii) Since $Y_{n, s}\left(\omega^{0}\right)$ converges as $n \rightarrow \infty$ for every $s \in T \backslash\{0\}$, Lemma 1 implies that $P^{\omega^{0}}$ exists. As for the "Markov chain" property, we will only give here the transition matrix $Q$ and its invariant probability measure $\pi$.

$$
\begin{aligned}
& Q=\left(\begin{array}{ll}
e^{2(\beta+J)}\left[e^{2(\beta+J)}+1\right]^{-1} & {\left[e^{2(\beta+J)}+1\right]^{-1}} \\
e^{2(\beta-J)}\left[e^{2(\beta-J)}+1\right]^{-1} & {\left[e^{2(\beta-J)}+1\right]^{-1}}
\end{array}\right)+1 \\
& +1 \quad-1 \\
& \pi=\left(\begin{array}{ll}
{\left[e^{2(\beta-J)}+e^{4 \beta}\right] /\left[e^{4 \beta}+2 e^{2(\beta-J)}+1\right]} \\
{\left[e^{2(\beta-J)}+1\right] /\left[e^{4 \beta}+2 e^{2(\beta-J)}+1\right]}
\end{array}\right)+1
\end{aligned}
$$

Now we will prove the equality $\mathscr{M} \cap \mathscr{G}(H, J)=\left\{P^{\omega^{+}}, P^{\omega^{-}}, P^{\omega^{0}}\right\}$ for all 
$H$ and $J$. At first, assume that $J \leqq \frac{1}{2} \log [(d+1) /(d-1)]$, then from Preston's theorem in $\S 3, \mathscr{G}(H, J)$ consists of a single point. Hence $P^{\omega^{+}}=P^{\omega^{-}}=P^{\omega^{0}}$. Thus in this case,

$$
\mathscr{M} \cap \mathscr{G}(H, J)=\left\{P^{\omega^{+}}, P^{\omega^{-}}, P^{\omega^{0}}\right\}\left(=\left\{P^{\omega^{+}}\right\}\right) .
$$

Let us fix $J>\frac{1}{2} \log [(d+1) /(d-1)]$ arbitrarily. If $|H|>d f(u)-u$, where $u$ is the positive solution of $f^{\prime}(u)=1 / d$, then from the same reason as above, $\mathscr{M} \cap \mathscr{G}(H, J)=\left\{P^{\omega^{+}}, P^{\omega^{-}}, P^{\omega^{0}}\right\}\left(=\left\{P^{\omega^{+}}\right\}\right)$. If $|H|<d f(u)-u$, then the equation (11) has exactly three solutions $\alpha<\beta<\gamma$, so $P^{\omega^{+}}, P^{\omega^{-}}$, $P^{\omega^{0}}$ are distinct. From Spitzer's theorem $\mathscr{M} \cap \mathscr{G}(H, J)$ has at most three elements, hence $\mathscr{M} \cap \mathscr{G}(H, J)=\left\{P^{\omega^{+}}, P^{\omega^{-}}, P^{\omega^{0}}\right\}$. If $H=d f(u)-u$, then $\alpha=\beta \neq \gamma$, i.e. $P^{\omega^{+}} \neq P^{\omega^{0}}=P^{\omega^{-}}$. If $H=u-d f(u)$, then $\alpha \neq \beta=\gamma$, i.e. $P^{\omega^{+}}$ $=P^{\omega^{0}} \neq P^{\omega^{-}}$. Anyway, if $|H|=d f(u)-u,\left\{P^{\omega^{+}}, P^{\omega^{-}}, P^{\omega^{0}}\right\}=\left\{P^{\omega^{+}}, P^{\omega^{-}}\right\}=$ the two point set. But we must note that Spitzer has shown in his paper [2] that the number of elements of $\mathscr{M} \cap \mathscr{G}(H, J)$ is equal to the number of $t$-solutions in $[0,1]$ of the equation

$$
t x^{2}+1-t=\left(\frac{1-t}{t}\right)^{1 / d} x(x y)^{-1 / d}
$$

with $x=e^{-2 J}, y=e^{-2 H}$. When $H$ varies monotonously from $-\infty$ to $\infty$, only the value of $y$ in (*) changes and it changes from 0 to $\infty$ monotonously. Hence the number of solutions of $(*)$ changes $1 \rightarrow 2 \rightarrow 3 \rightarrow 2 \rightarrow 1$ as $H$ increases from $-\infty$ to $\infty$. This means that the number of elements of $\mathscr{M} \cap \mathscr{G}(H, J)$ is equal to 2 if $|H|=d f(u)-u$. Thus we have $\mathscr{M} \cap \mathscr{G}(H$, $J)=\left\{P^{\omega^{+}}, P^{\omega^{-}}, P^{\omega^{0}}\right\}$ for all $H$ and $J$.

Q.E.D.

Remark. Spitzer [2] has claimed that a "Markov chain" is an extremal Gibbs state, but it is not true. In fact T. Kamae [4] has shown that if $H=0$, then $\mathscr{B}_{\infty}=\bigcap_{V \text {; f inite }} \mathscr{B}_{V^{c}}$ is not trivial with respect to $P^{\omega^{0}}$. We will briefly quote it here. Put $f_{n}(\sigma)=\sum_{t \in \partial V_{n}} \sigma(t)$. Then $f_{n}(\sigma)$ is $\mathscr{B}_{V_{n}^{c_{-}}}$ -measurable and $\left\|f_{n}\right\|_{2, \omega^{0}}^{2}=E^{\omega^{0}}\left[f_{n}^{2}\right] \leqq(d+1) d^{n-1} \sum_{k=0}^{n}\left[d(2 p-1)^{2}\right]^{k},\left\langle f_{n}\right\rangle_{\omega^{0}}$ $=E^{\omega^{0}}\left(f_{n}\right)=0, \quad\left\langle f_{n}, X_{0}>{ }_{\omega^{0}}=E^{\omega^{0}}\left[f_{n} X_{0}\right]=(d+1) d^{n-1}(2 p-1)^{n}, \quad\right.$ where $p$ $=Q(+1,+1)=Q(-1,-1)=e^{2 J}\left[e^{2 J}+1\right]^{-1}$. Hence if $d(2 p-1)^{2}>1$,

$$
<f_{n} /\left\|f_{n}\right\|_{2, \omega^{0}}, X_{0}>_{\omega^{0}} \geqq C(d, p)>0 \quad \text { for all } n \geqq 1 \text {, }
$$


where $C(d, p)$ is a positive constant depending only on $d$ and $p$. But (**) implies that

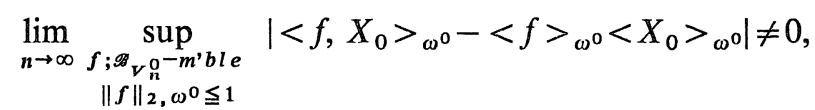

which means that $\mathscr{B}_{\infty}$ is non-trivial with respect to $P^{\omega^{0}}$.

\section{§5. Small Order Perturbations}

Let us fix $k, 1 \leqq k \leqq d-1$, and a connected subgraph $\tilde{T} \subset T$ such that (i) $\tilde{T} \supset V_{1}$ and (ii) every $t \in \tilde{T} \backslash\{0\}$ has exactly $k+1$ neighbours in $\tilde{T}$. Then it is easy to see that $\lim _{n \rightarrow \infty} \sharp\left(\tilde{T} \cap \partial V_{n}\right) / \#\left(\partial V_{n}\right)=0$. Define $\omega^{k} \in \Sigma$ by

$$
\omega^{k}(t)=\left\{\begin{array}{lll}
+1 & \text { if } & t \in \widetilde{T} \\
-1 & \text { if } & t \in T \backslash \widetilde{T}
\end{array}\right.
$$

Lemma 2. $P^{\omega^{k}}=\mathrm{w}-\lim _{n \rightarrow \infty} P_{V_{n}}^{\omega^{k}}$ exists.

Proof. Note that

$$
Y_{n, i}\left(\omega^{k}\right)=k f\left(Y_{n-1, i}\left(\omega^{k}\right)\right)+(d-k) f\left(Y_{n-1, i}\left(\omega^{-}\right)\right)+H
$$

for every $1 \leqq i \leqq d+1$ and $n \geqq 2$, where $f(x)$ and $Y_{n, i}(\omega)$ are defined by (10) and (15) respectively. Moreover, we have

$$
Y_{n, s}\left(\omega^{k}\right)=\left\{\begin{array}{lll}
Y_{n-|s|+1,1}\left(\omega^{k}\right) & \text { if } & s \in \tilde{T} \backslash\{0\}, \\
Y_{n-|s|+1}\left(\omega^{-}\right) & \text {if } & s \in T \backslash \tilde{T}
\end{array}\right.
$$

Combining Lemma 1 and (16), we have only to show that $Y_{n, 1}\left(\omega^{k}\right)$ converges as $n \rightarrow \infty$.

Let us define a function $F_{\lambda}^{H}(x)$ by

$$
F_{\lambda}^{H}(x)=k f(x)-(d-k) f(\lambda)+H \text {. }
$$

Since $Y_{n}\left(\omega^{-}\right) / \alpha$ as $n \rightarrow \infty$, for any given $\varepsilon>0$ there is $n_{\varepsilon}>0$ such that

$$
F_{-\alpha}^{H}\left(Y_{n-1,1}\left(\omega^{k}\right)\right)>Y_{n, 1}\left(\omega^{k}\right)>F_{-\alpha}^{H}\left(Y_{n-1,1}\left(\omega^{k}\right)\right)-\varepsilon
$$

for all $n \geqq n_{\varepsilon}$. The equation $F_{\lambda}^{H}(x)=x$ has at least one and at most 
three solutions. We will only consider here the case when the equation $F_{-\alpha}^{H}(x)=x$ has three solutions, say $\delta_{1}<\delta_{2}<\delta_{3}$. (In other cases, the proof will be easier.) Choose $\varepsilon>0$ so small that the equation $F_{-\alpha}^{H}(x)-\varepsilon=x$ has also three solutions $\delta_{1}^{\varepsilon}<\delta_{2}^{\varepsilon}<\delta_{3}^{\varepsilon}$. Note that $\delta_{1}^{\varepsilon} / \delta_{1}, \delta_{2}^{\varepsilon} \backslash \delta_{2}, \delta_{3}^{\varepsilon} / \delta_{3}$ as $\varepsilon \rightarrow 0$. We will prove that (i) if $\lim _{n \rightarrow \infty} Y_{n, 1}\left(\omega^{k}\right)<\delta_{2}$ then $\lim _{n \rightarrow \infty} Y_{n, 1}\left(\omega^{k}\right)=\delta_{1}$, (ii) if $\varlimsup_{n \rightarrow \infty} Y_{n, 1}\left(\omega^{k}\right)>\delta_{2}$ then $\lim _{n \rightarrow \infty} Y_{n, 1}\left(\omega^{k}\right)=\delta_{3}$. Assume first that $\lim _{n \rightarrow \infty} Y_{n, 1}\left(\omega^{k}\right)$ $<\delta_{2}$. Then there exists $n>n_{\varepsilon}$ such that $Y_{n, 1}\left(\omega^{k}\right)<\delta_{2}$. Using (17) we get $\delta_{1}^{\varepsilon} \leqq \lim _{n \rightarrow \infty} Y_{n, 1}\left(\omega^{k}\right) \leqq \varlimsup_{n \rightarrow \infty} Y_{n, 1}\left(\omega^{k}\right) \leqq \delta_{1}$. Letting $\varepsilon \searrow 0$ we obtain (i). Next, we assume $\varlimsup_{n \rightarrow \infty} Y_{n, 1}^{n \rightarrow \infty}\left(\omega^{k}\right)>\delta_{2}+\theta$ for some $\theta>0$. Choosing $\varepsilon>0$ so small that $\delta_{2}^{\varepsilon}<\delta_{2}+\theta$, we can conclude from (17) that $\delta_{3}^{\varepsilon} \leqq \lim _{n \rightarrow \infty} Y_{n, 1}\left(\omega^{k}\right) \leqq \varlimsup_{n \rightarrow \infty} Y_{n, 1}\left(\omega^{k}\right)$ $\leqq \delta_{3}$. This means (ii).

Theorem 2. If $H=0, k>d / 2+1$ and if the equation

$$
F_{d J}^{0}(x)=x
$$

has more than one solutions, then

$$
P^{\omega^{k}}(\sigma(0)=1)=\frac{1}{1+\left[\operatorname{ch}\left(J-\delta_{3}\right) / \operatorname{ch}\left(J+\delta_{3}\right)\right]^{d+1}}>1 / 2
$$

Remark. If $J \geqq \frac{k \log 2}{2(2 k-d+1)}$, then $k f(J)-(d-k) J \geqq J$. Since $|f(x)|<J$ for all $x$, we have $F_{d J}^{0}(J)>k f(J)-(d-k) J \geqq J$. This implies that (17) has three solutions.

Proof of Theorem 2. Let $\alpha<\beta<\gamma$ be the solutions of (11) as before. Since $H=0$ we have $\beta=0$ and $\alpha=-\gamma$. Noting $\gamma=d f(\gamma)<d J$, we have $F_{\gamma}^{0}(x)>F_{d J}^{0}(x)$ for all $x \in(-\infty, \infty)$. Hence the assumption of the theorem implies that the equation $F_{\gamma}^{0}(x)=x$ has three solutions $\delta_{1}<\delta_{2}<\delta_{3}$. Since $\delta_{3}<\gamma$ and $F_{\gamma}^{0}(x)<k f(x)-(d-k) f(x)=(2 k-d) f(x)$ for $0<x<\gamma$, the equation $(2 k-d) f(x)=x$ has three solutions $\alpha^{\prime}<\beta^{\prime}<\gamma^{\prime}$, and $\delta_{3}<\gamma^{\prime}=$ $(2 k-d) f\left(\gamma^{\prime}\right)<(2 k-d) J=Y_{1,1}\left(\omega^{k}\right)$.

On the other hand we have

$$
F_{d J}^{0}\left(Y_{n-1,1}\left(\omega^{k}\right)\right) \leqq Y_{n, 1}\left(\omega^{k}\right) \leqq F_{\gamma}^{0}\left(Y_{n-1,1}\left(\omega^{k}\right)\right)
$$

for all $n \geqq 2$ from $(17)$ and $Y_{n-1}\left(\omega^{-}\right) \geqq-d J$. From Lemma 2, $\lim _{n \rightarrow \infty} Y_{n, 1}\left(\omega^{k}\right)$ 
exists and is a solution of the equation $F_{\gamma}^{0}(x)=x$. But from (19) and $Y_{1,1}\left(\omega^{k}\right)>\delta_{3}$ we have $\delta_{2}<\delta_{3}^{\prime} \leqq \lim _{n \rightarrow \infty} Y_{n, 1}\left(\omega^{k}\right) \leqq \delta_{3}$, where $\delta_{1}^{\prime} \leqq \delta_{2}^{\prime} \leqq \delta_{3}^{\prime}$ are the solutions of the equation $(18)$. Hence $\lim _{n \rightarrow \infty} Y_{n, 1}\left(\omega^{k}\right)=\delta_{3}>0$. From this and (6), we obtain the conclusion of the theorem.

Q.E.D.

\section{§6. Extremal Gibbs States}

In this section, we will give several extremal points of $\mathscr{G}(H, J)$ as the limiting Gibbs states. In order to do so, we need the following theorem which is stated under a more general framework in [5].

Theorem (Miyamoto [5]). Let $P \in \mathscr{G}(H, J)$, then $P$ is an extremal point of $\mathscr{G}(H, J)$ iff

$$
P^{\omega}=\mathrm{w}-\lim _{n \rightarrow \infty} P_{V_{n}}^{\omega}=P \quad \text { a.s. } \quad(P)
$$

Lemma 3. The condition (20) for $P \in \mathscr{G}(H, J)$ is equivalent to

$$
\lim _{n \rightarrow \infty} R_{n, s}(\omega)=r_{s} \quad \text { a.s. } \quad(P)
$$

for each $s \in T$ with $|s| \geqq N$ for some $N>0$, where $r_{s}$ is a constant depending only on s.

Proof. Assume (20). Then for every pair $t, s \in T$ with $t \rightarrow s$, we have

$$
\lim _{n \rightarrow \infty} P_{V_{n}}^{\omega}(\sigma(s)=+1 \mid \sigma(t)=+1)=P(\sigma(s)=+1 \mid \sigma(t)=+1) \quad \text { a.s. } \quad(P) .
$$

On the other hand we have

$$
P_{V_{n}}^{\omega}(\sigma(s)=+1 \mid \sigma(t)=+1)=e^{2 J} R_{n, s}(\omega) /\left(e^{2 J} R_{n, s}(\omega)+1\right) .
$$

Hence we get (21) for all $s \in T \backslash\{0\}$. Conversely assuming (21) we get (20) from (7), (8) and (3).

Q.E.D.

Corollary. If $P \in \mathscr{G}(H, J)$, then the limit $r_{s}(\omega)=\lim _{n \rightarrow \infty} R_{n, s}(\omega)$ exists almost surely with respect to $P$ for every $s \in T \backslash\{0\}$.

Theorem 3. Under the assumptions of Theorem 2, $P^{\omega^{k}}$ is an extremal point of $\mathscr{G}(0, J)$. 
Proof. $\left.1^{\circ}\right)$ For any $s \in T \backslash \tilde{T}$ and $t$ with $t \rightarrow s$, we have $P^{\omega^{k}}(A \mid \sigma(t)$ $=\varepsilon)=P^{\omega^{-}}(A \mid \sigma(t)=\varepsilon)$ for all $A \in \mathscr{B}_{T s}$, where $\varepsilon=+1$ or -1 . Hence we get

$$
\begin{aligned}
P^{\omega^{k}} & \left(\lim _{n \rightarrow \infty} R_{n, s}(\sigma)=e^{2 \alpha}\right) \\
= & P^{\omega^{k}}(\sigma(t)=+1) P^{\omega^{-}}\left(\lim _{n \rightarrow \infty} R_{n, s}(\sigma)=e^{2 \alpha} \mid \sigma(t)=+1\right) \\
& \quad+P^{\omega^{k}}(\sigma(t)=-1) P^{\omega^{-}}\left(\lim _{n \rightarrow \infty} R_{n, s}(\sigma)=e^{2 \alpha} \mid \sigma(t)=-1\right) \\
= & 1
\end{aligned}
$$

$2^{\circ}$ ) Defining $G(x)$ by $G(x)=\left(1+e^{2 J} x\right) /\left(e^{2 J}+x\right)$, we have from (7) and $1^{\circ}$ ),

$$
\begin{aligned}
r_{t}(\omega) & =\lim _{n \rightarrow \infty} R_{n, t}(\omega)=\prod_{\substack{u \in \widetilde{T} \\
t \rightarrow u}} G\left(r_{u}(\omega)\right) \prod_{\substack{v \in T \backslash \widetilde{T} \\
t \rightarrow v}} G\left(r_{v}(\omega)\right) \\
& =\prod_{u \in \widetilde{T}} G\left(r_{u}(\omega)\right) G\left(e^{2 \alpha}\right)^{d-k} \\
& \leqq G\left(e^{2 \gamma}\right)^{2 k-d}
\end{aligned}
$$

almost surely with respect to $P^{\omega^{k}}$, for all $t \in \tilde{T} \backslash\{0\}$. (Note that $r_{u}(\omega)$ $\leqq r_{u}\left(\omega^{+}\right)=e^{2 \gamma}$ for all $\omega \in \Sigma, u \in T \backslash\{0\}$.) Hence we have

$$
y_{t}(\omega)=\lim _{n \rightarrow \infty} Y_{n, t}(\omega)=\sum_{\substack{u \in T \\ t \overrightarrow{1} u}} f\left(y_{u}(\omega)\right)-(d-k) f(\gamma)
$$

almost surely with respect to $P^{\omega^{k}}$, and

$$
y_{t}(\omega) \leqq(2 k-d) f(\gamma)=F_{\gamma}^{0}(\gamma) \quad \text { a.s. } \quad\left(P^{\omega^{k}}\right),
$$

for every $t \in \tilde{T} \backslash\{0\}$. Using (23) and (24) we get

$$
y_{t}(\omega) \leqq k f\left(F_{\gamma}^{0}(\gamma)\right)-(d-k) f(\gamma)=F_{\gamma}^{0}\left(F_{\gamma}^{0}(\gamma)\right) \quad \text { a.s. } \quad\left(P^{\omega^{k}}\right),
$$

and inductively,

$$
y_{t}(\omega) \leqq{\widehat{F_{\gamma}^{0}\left(F _ { \gamma } ^ { 0 } \left(\cdots \left(F_{\gamma}^{0}\right.\right.\right.}}_{(\gamma)) \cdots)}^{n} \quad \text { a.s. } \quad\left(P^{\omega^{k}}\right)
$$

for every $t \in T \backslash\{0\}$ and $n \geqq 1$. This implies

$$
y_{t}(\omega) \leqq \delta_{3} \quad \text { a.s. } \quad\left(P^{\omega^{k}}\right)
$$


for every $t \leqq T \backslash\{0\}$ because $\delta_{3}<\gamma$, where $\delta_{3}$ is the largest solution of $F_{\gamma}^{0}(x)=x$.

$\left.3^{\circ}\right)$ Define $A_{t}=\left\{\omega \in \Sigma ; y_{t}(\omega)<\delta_{3}\right\}$ for $t \in \tilde{T} \backslash\{0\}$. If $t, t^{\prime} \in \widetilde{T}$ are such that $t \rightarrow t^{\prime}$, (23) and (24) imply $A_{t^{\prime}} \subset A_{t}$. Hence $A_{t} \subset A_{i}$ if $i \rightarrow t \in \widetilde{T}$. Since

$$
\begin{aligned}
& P^{\omega^{k}}\left(\sigma(0)=+1 \mid \bigcap_{n} \mathscr{B} V_{n}^{c}\right)(\omega)=\lim _{n \rightarrow \infty} P_{V_{n}}^{\omega}(\sigma(0)=+1) \\
& \quad=\left[\prod_{i=1}^{d+1} G\left(r_{i}(\omega)\right)\right] /\left[1+\prod_{i=1}^{d+1} G\left(r_{i}(\omega)\right)\right] \leqq G\left(e^{2 \delta_{3}}\right)^{d+1} /\left[1+G\left(e^{2 \delta_{3}}\right)^{d+1}\right] \\
& \quad=P^{\omega^{k}}(\sigma(0)=+1) \quad \text { a.s. } \quad\left(P^{\omega^{k}}\right),
\end{aligned}
$$

we have

$$
P^{\omega^{k}}\left(y_{i}(\sigma)=\delta_{3}, 1 \leqq i \leqq d+1\right)=1,
$$

which means $P^{\omega^{k}}\left(A_{i}\right)=0$ and so $P^{\omega^{k}}\left(A_{t}\right)=0$, i.e. $\lim _{n \rightarrow \infty} R_{n, t}(\omega)=e^{2 \delta_{3}}$ a.s. $\left(P^{\omega^{k}}\right)$ for all $t \in \widetilde{T}$. Combining this with $1^{\circ}$ ), Lemma 3 proves the theorem.

Q.E.D.

Let us fix any $n \geqq 1$ and write the points of $\partial V_{n}$ by $u_{1}, u_{2}, \ldots, u_{m(n)}$ where $m(n)=(1+d) d^{n}$. Then we have

Theorem 4. Assume that for some $n \geqq 1, P^{\omega_{1}}, P^{\omega_{2}}, \ldots, P^{\omega_{m(n)}}$ are extremal points of $\mathscr{G}(H, J)$. If we define a new boundary condition $\tilde{\omega} b y$

$$
\tilde{\omega}(t)=\omega_{j}(t) \quad \text { if } \quad t \in T_{u_{j}} \quad 1 \leqq j \leqq m(n)
$$

then $P^{\tilde{\omega}}$ is also an extremal point of $\mathscr{G}(H, J)$.

Proof. Using the analogous argument as in the proof of the preceeding theorem, we obtain that if $u_{j} \rightarrow t$ then $r_{t}(\omega)=r_{t}\left(\omega_{j}\right)$ a.s. $\left(P^{\tilde{\omega}}\right)$. Hence from Lemma 3, we get the conclusion.

Q.E.D.

Acknowledgement. The author would like to thank Prof. H. Totoki for his many advices and Prof. T. Shiga, Prof. M. Miyamoto and Prof. T. Kanuae and Mr. T. Ohno for many discussions with them. 


\section{References}

[1] Preston, C. J., Gibbs states on countable sets, Cambridge University Press 1974.

[2] Spitzer, F., Markov random fields on an infinite tree, Ann. Probability, 3 (1975), 387-398.

[3] Katsura, S., Phase transitions of ferromagnetic Ising models, Suuri Kagaku No. 148, October, 1975. (in Japanese)

[4] Kamae, T., Private communication.

[5] Miyamoto, M., Martin-Dynkin boundaries of random fields, Comm. Math. Phys., 36, 1974. 